\title{
USO DA FOTOGRAFIA NO PROCESSO DO CUIDAR: TENDÊNCIAS DAS AÇÕES DE ENFERMAGEM
}

\author{
USE OF PHOTOGRAPHY IN THE PROCESS OF CARING: \\ TRENDS IN NURSING ACTIONS
}

\section{USO DE LA FOTOGRAFÍA EN EL PROCESO DEL CUIDAR: TENDENCIAS DE ACCIONES DE ENFERMERÍA}

\author{
Marli Teresinha Gimeniz Galvão* \\ Herta de Oliveira Alexandre* \\ Patrícia Bernardo Dantas ${ }^{* * *}$ \\ IVANa CRISTINA VieIRA DE LIMA ${ }^{* * * *}$ \\ EMELINe MOURA LOPES ${ }^{* * * *}$
}

\begin{abstract}
RESUMO
Objetivou-se analisar, com base em evidências científicas, as tendências das ações de enfermagem mediadas pelo uso da fotografia. Realizou-se uma revisão integrativa, nas bases de dados LILACS, PubMed e CINAHL, por meio dos descritores nursing care e photography. Foram selecionados sete artigos em inglês, português e espanhol; sem restrição quanto ao ano de publicação. Os estudos apontam para uma maior utilização da fotografia no cuidado com pacientes com doença mental, na evolução de feridas e como forma de aprimorar a comunicação entre equipe de saúde e clientes. Conclui-se que a fotografia é um recurso disponível para avaliar o cuidado de enfermagem, decorrente das inúmeras informações produzidas mediante os registros visuais, possibilitando ao enfermeiro planejar sua assistência de forma mais precisa. Contudo, esta técnica ainda é pouco utilizada, especialmente no Brasil.
\end{abstract}

Palavras chave: Fotografia, cuidados de Enfermagem, Enfermagem.

\begin{abstract}
This study aimed to analyze, based on scientific evidence, trends of nursing actions through the use of photography. Was performed an integrative review, whose articles were selected in the databases LILACS, CINAHL and PubMed, using the keywords: "nursing care" and "photography". Were selected seven articles in english, portuguese and spanish, without restriction as to the year of publication. The studies point out to greater use of photography in the care of patients with mental, illness in the evolution of wounds and as a way to improve communication between health staff and customers. We conclude that the use of photography is of great value in nursing care and the amount of information that can be obtained through visual records are countless, al-

\footnotetext{
* Enfermeira. Professora do Departamento de Enfermagem Universidade Federal do Ceará. Fortaleza. Brasil. Email: marligalvao@gmail.com

${ }_{* *}^{*}$ Enfermeira. Universidade Federal do Ceará. Fortaleza. Brasil. Email: herta.oliveira@yahoo.com.br

${ }^{* * *}$ Enfermeira. Universidade Federal do Ceará. Fortaleza. Brasil. Email: patricia_dantas1703@hotmail.com

${ }^{* * * *}$ Enfermeira. Universidade Federal do Ceará. Fortaleza. Brasil Email: ivanacristinalima@gmail.com

${ }^{* * * * *}$ Enfermeira. Universidade Federal do Ceará. Fortaleza. Brasil. Email: emelinepet@yahoo.com.br
} 
lowing nurses to plan your assistance more accurately. However, this technique is not widely used, especially in Brazil.

Key words: Photography, Nursing care, Nursing.

\section{RESUMEN}

Este estudio tuvo como objetivo analizar, con base en la evidencia científica, la evolución de las acciones de enfermería a través del uso de la fotografía. Se realizó una revisión integradora, cuyos artículos fueron seleccionados en las bases de datos LILACS, CINAHL y PubMed, utilizando las palabras clave: "cuidado de enfermería" y "fotografía". Fueron seleccionados siete artículos en inglés, portugués y español, sin restricción en cuanto al año de publicación. Los estudios apuntan a un mayor uso de la fotografía, en el cuidado de pacientes con enfermedades mentales, en la evolución de las heridas y como una forma de mejorar la comunicación entre el personal sanitario y los clientes. Concluímos que el uso de la fotografía es de gran valor en la atención de enfermería y la cantidad de información que se puede obtener a través de registros visuales son innumerables, lo que permite a los enfermeros planear su asistencia con mayor precisión. Sin embargo, esta técnica no se utiliza ampliamente, especialmente en Brasil.

Palabras clave: Fotografia, cuidados de Enfermería, Enfermería.

Fecha de recepción: 29/11/12 Fecha aceptación: 16/10/13

\section{INTRODUÇÃO}

A enfermagem, enquanto ciência, dedica-se ao desenvolvimento de teorias e metodologias para o aperfeiçoamento das ações desenvolvidas. A profissão converge os conceitos de ciência-arte e tecnologia para a prática assistencial e de ensino em diferentes áreas tecnológicas de estudo (1). Nessa perspectiva, é importante buscar novas estratégias para mediar e avaliar o cuidado em saúde.

Sob essa ideia, a fotografia traz um novo modo de olhar, fixando um instante, possibilitando a visibilidade sobre detalhes ou um pequeno gesto que, de outro modo, poderia ser imperceptível (2). A imagem mediada pela fotografia é um recurso pouco explorado na assistência de enfermagem.

Historicamente, a inserção da fotografia nas ciências ocorreu em função do desenvolvimento de doenças mentais a partir da segunda metade do século XIX com pacientes de hospitais e asilos da França e da Inglaterra
(3). A imagem pode auxiliar as ciências em seu esforço para uma melhor apreensão da realidade do mundo (4), sendo a fotografia incorporada às práticas médicas e científicas como um instrumento preciso e absoluto de observação (3).

A fotografia contribui decisivamente na realização de pesquisas teóricas, manifestações artístico-culturais e como coadjuvante eficaz em inúmeras descobertas científicotecnológicas. Contribui para a ciência, pois representa uma sequência qualificada de informação que não pode ser obtida de nenhuma outra forma (5).

$\mathrm{Na}$ área da saúde, sua utilização permite ilustrar um evento, bem como seu uso pode ser reconhecido por auxiliar a prática no que concerne ao diagnóstico e tratamento de doenças $(2,6)$.

A fotografia tem sido utilizada para a compreensão de experiências vividas por pacientes, para pesquisa, comparações da cicatrização de feridas e no estudo da comunicação entre profissional e cliente (7-9). As 
imagens comprovam a realização de avaliação da saúde, de planejamento de cuidados específicos e atividades de cuidados de qualidade (10).

Nas análises sobre a prática de enfermagem no tratamento de feridas, onde mais se visualiza o uso de fotografia, ainda se fazem necessários estudos sobre sua utilização e a avaliação de técnicas apropriadas, visto que a sistematização do processo fotográfico pode auxiliar a prática de enfermagem nos cuidados de pacientes portadores de feridas (8).

A investigação do uso da fotografia no cuidado de enfermagem pode contribuir para uso desse método de registro em saúde, fornecendo ferramentas para avaliação e registro do trabalho do enfermeiro e consequentemente melhorias na qualidade dos serviços prestados.

Desse modo, justifica-se a proposta do presente estudo, cuja contribuição original consiste em analisar, com base em evidências científicas, as tendências das ações de enfermagem que utilizam a fotografia.

\section{MÉTODOS}

Esta pesquisa teve como percurso metodológico uma revisão integrativa. Trata-se da mais ampla abordagem metodológica referente às revisões, permitindo a inclusão de estudos experimentais e não-experimentais para uma compreensão completa do fenômeno analisado. Consiste ainda em um método de pesquisa utilizado para reunir e analisar as evidências disponíveis e fornecer as intervenções que poderiam proporcionar um cuidado mais efetivo e de melhor custo/ benefício (11).

O estudo foi operacionalizado seguindose as etapas: identificação do tema e seleção da questão de pesquisa, estabelecimento de critérios para inclusão e exclusão de estudos, amostragem ou busca na literatura, definição das informações a serem extraídas dos estudos selecionados, avaliação dos estudos incluídos na revisão integrativa, interpretação dos resultados e apresentação da revisão (12).

A elaboração desta revisão foi definida a partir da seguinte pergunta norteadora: Qual as tendências das ações de enfermagem mediadas pelo uso da fotografia?

Este estudo não teve recorte temporal, em função da escassez de dados. Entretanto foram observados trabalhos publicados até dezembro de 2011. O desenvolvimento do estudo foi efetivado nos meses de fevereiro e março de 2012.

Nesta pesquisa foram adotados como critérios de inclusão para busca dos artigos os seguintes parâmetros: retratar cuidados procedimentos ou intervenções de enfermagem utilizando o recurso da fotografia; publicados em inglês, português e espanhol; sem restrição quanto ao ano de publicação.

Como critérios de exclusão, estabeleceram-se os seguintes limites: impossibilidade de obtenção do artigo na íntegra, revisões de literatura, apresentação sob o formato de dissertação, tese, capítulo de livro, livro, editorial, resenha, comentário ou crítica e inadequação à questão norteadora do estudo.

Para a seleção dos artigos incluídos na revisão foi realizada busca nas bases de dados LILACS (Literatura Latino Americana e do Caribe em Ciências), PubMed (Public/Editora Medline) e CINAHL (Índice Acumulado para a Enfermagem e Literatura Allied Health). Foram utilizados para busca os descritores controlados: "nursing care" e "photography", separadas pelo operador booleano and.

Inicialmente foram identificados 329 estudos. Empreendeu-se à leitura dos resumos e, de acordo com os critérios de inclusão e exclusão, resultaram 15 artigos na pré-seleção. Depois da leitura integral destes, oito foram excluídos por não se adequarem ao tema da questão norteadora. Assim, sete estudos foram selecionados e analisados na íntegra, sendo quatro localizados no CINAHL, 
dois no PubMed e um no LILACS.

Para o desenvolvimento da apresentação dos resultados realizou-se a leitura crítica dos trabalhos, destacando os seguintes aspectos: base de dados, título, periódico, ano, autores, objetivos, resultados e conclusões dos estudos, por meio de instrumento de coleta de dados elaborado pelos autores.

\section{RESULTADOS}

Nesta revisão analisaram-se os sete artigos que atenderam aos critérios de inclusão previamente estabelecidos constatou-se que seis foram publicados em inglês e um em português (Tabela 1).

Tabela 1. Distribuição dos estudos, segundo títulos, periódico, ano, autores e objetivos. Fortaleza. Março 2012.

\begin{tabular}{|c|c|c|c|c|}
\hline Título & Periódico & Ano & Autor(es) & Objetivo \\
\hline $\begin{array}{l}\text { Pictures of a Client's Si- } \\
\text { lent Distress. }\end{array}$ & Creative Nursing & 2011 & Felicilda RF. & $\begin{array}{l}\text { Descrever como as imagens, tanto } \\
\text { mentais quanto impresso, podem } \\
\text { ser utilizadas pelos enfermeiros } \\
\text { para abrir caminhos de comunica- } \\
\text { ção de cura e cuidados de enferma- } \\
\text { gem holística. }\end{array}$ \\
\hline $\begin{array}{l}\text { Moral learning in psy- } \\
\text { chiatric Rehabilitation }\end{array}$ & Nursing Ethics & 2011 & $\begin{array}{l}\text { Sitvast JE, Widdershoven } \\
\text { GAM, Abma TB. }\end{array}$ & $\begin{array}{l}\text { Ilustrar a aprendizagem moral de } \\
\text { pessoas com deficiência psiquiátri- } \\
\text { ca que participaram de uma inter- } \\
\text { venção de enfermagem, através de } \\
\text { foto instrumento. }\end{array}$ \\
\hline $\begin{array}{l}\text { Cuidados de enferma- } \\
\text { gem e segurança do } \\
\text { paciente: visualizando a } \\
\text { organização, acondicio- } \\
\text { namento e distribuição } \\
\text { de medicamentos com } \\
\text { método de pesquisa fo- } \\
\text { tográfica. }\end{array}$ & $\begin{array}{l}\text { Revista Latino-A- } \\
\text { mericana de Enfer- } \\
\text { magem }\end{array}$ & 2010 & $\begin{array}{l}\text { Raduenz AC, Hoffmann } \\
\text { P, Radunz V, Dal Sas- } \\
\text { so GTM, Maliska ICM, } \\
\text { Marck PB. }\end{array}$ & $\begin{array}{l}\text { Descrever os achados seleciona- } \\
\text { dos de relatórios de enfermeiros e } \\
\text { acadêmicos de enfermagem acer- } \\
\text { ca da organização, distribuição e } \\
\text { acondicionamento de medicações, } \\
\text { numa unidade de clínica médica } \\
\text { de um hospital público de ensino, } \\
\text { no Brasil. }\end{array}$ \\
\hline $\begin{array}{l}\text { A nurse-led intensive } \\
\text { care after-care pro- } \\
\text { gramme-development, } \\
\text { experiences and prelim- } \\
\text { inary evaluation. }\end{array}$ & $\begin{array}{l}\text { Nursing in Critical } \\
\text { Care }\end{array}$ & 2009 & $\begin{array}{l}\text { Samuelson KA, Corrigan } \\
\text { I. }\end{array}$ & $\begin{array}{l}\text { Estabelecer um cuidado intensivo } \\
\text { após programa de tratamento e } \\
\text { realizar uma avaliação preliminar } \\
\text { do serviço de acompanhamento a } \\
\text { partir das perspectivas dos pacien- } \\
\text { tes e familiares. }\end{array}$ \\
\hline $\begin{array}{l}\text { An Ethnographic Ap- } \\
\text { proach to Interpreting } \\
\text { a Mental Illness Photo- } \\
\text { voice Exhibit }\end{array}$ & $\begin{array}{l}\text { Archives of Psy- } \\
\text { chiatric Nursing }\end{array}$ & 2009 & $\begin{array}{l}\text { Fleming J, Mahoney J, } \\
\text { Carlson E, Engebretson J. }\end{array}$ & $\begin{array}{l}\text { Explorar o uso de fotovoz como } \\
\text { uma inovadora metodologia para a } \\
\text { compreensão de aspectos da expe- } \\
\text { riência da doença mental. }\end{array}$ \\
\hline $\begin{array}{l}\text { The importance of } \\
\text { wound documentation } \\
\text { and classification. }\end{array}$ & $\begin{array}{l}\text { British Journal of } \\
\text { Nursing }\end{array}$ & 1999 & Russell L. & $\begin{array}{l}\text { Relatar a importância da docu- } \\
\text { mentação de feridas. }\end{array}$ \\
\hline Photography in nursing. & $\begin{array}{l}\text { American Journal } \\
\text { of Nursing }\end{array}$ & 1998 & Krainski M. & $\begin{array}{l}\text { Descrever a utilização de fotogra- } \\
\text { fias como um procedimento de } \\
\text { tratamento de feridas. }\end{array}$ \\
\hline
\end{tabular}


Em relação ao país de origem das publicações, dois foram desenvolvidos nos Estados Unidos da América, um na Inglaterra, um na Suécia, um nas Filipinas, um no Brasil e um na Holanda.

Os artigos foram publicados, em sua grande maioria, em periódicos da área de concentração da enfermagem. Apenas um artigo foi publicado em um periódico multidisciplinar. Este resultado justifica-se pelo fato de ter sido incluído o descritor "nursing" para busca de artigos, em que as publicações concentram-se em periódicos da própria área.

Observou-se que, em relação ao período da publicação, o ano precoce foi de 1998, com um artigo. Entretanto foram observadas publicações de um estudo em 1999, dois em 2009, um em 2010 e dois artigos em 2011.

Percebeu-se linearidade quanto às publicações de artigos que envolvem ações de enfermagem com uso de fotografias em relação ao ano, significando o reconhecimento destes profissionais à necessidade de utilização destes recursos e aos benefícios destes para a prática da enfermagem.

A Tabela 1 descreve o foco de cada estudo acerca do cuidado de enfermagem por meio do uso da fotografia.

O estudo intitulado "Pictures of a Client's Silent Distress", cujo objetivo foi descrever como as imagens podem ser utilizadas pelos enfermeiros para abrir caminhos de comunicação de cura e cuidados de enfermagem holística, evidenciou que o uso da fotografia na assistência proposta foi útil ao alertar o enfermeiro para os problemas psicossocio-espirituais apresentados por seus clientes. O estudo mostrou que a fotografia tornou-se um ponto de partida para uma nova avaliação da angústia intrapessoal e interpessoal que o cliente pode ter experimentado durante sua doença. O compartilhamento de fotos pode ser uma versão modificada da terapia narrativa que é eficaz no cuidado para clientes com angústias internas. Em seus resultados, o estudo aponta que narrativas podem ser ainda mais terapêuticas se combinadas com o compartilhamento de fotos, pois a representação visual de eventos tende a manter-se na memória (9).

Verifica-se, de forma subentendida, a comunicação estabelecida entre profissional e cliente, por meio do uso da fotografia. Ao se falar sobre comunicação, comumente a primeira ideia remete à comunicação verbal. Contudo, quando se fala de cuidar, é importante citar que, além da comunicação verbal, há outros métodos de expressar mensagens e sentimentos, onde pode-se citar a fotografia na captação dessas mensagens.

Os artigos "Moral learning in psychiatric rehabilitation" " "An Ethnographic Approach to Interpreting a Mental Illness Photovoice Exhibit" trazem à tona exemplo de uma das primeiras utilizações, que foi junto a pacientes com alterações mentais $(13,14)$.

O primeiro descreveu o uso da fotografia por pessoas com problemas psiquiátricos como forma de retratar a importância de suas vidas e desejos futuros; e o segundo investigou a experiência da doença mental e compreender os aspectos, respectivamente. Ambos os estudos evidenciaram que o uso da fotografia foi capaz de despertar o interesse dos clientes em compartilhar suas dúvidas interiores e anseios com a equipe de saúde e outras pessoas no grupo de reabilitação, e que desempenhou um papel importante no processo de tratamento. Acontece que pessoas com deficiência psiquiátrica não costumam expressar alguns aspectos de sua individualidade. O sofrimento isola as pessoas e tende a contê-los em si mesmos. Sentimentos de vergonha e perda podem colidir com capacidade de alguém de dar voz a outras vertentes da identidade do que aquelas que são normalmente expressas. Ao exporem suas histórias pessoais evidencia-se que os seus narradores são dignos de serem vistos e ouvidos, e que eles não precisam se envergonhar e retirar-se do contato. Isto é essencial para a reabilitação, porque convida alguém para vir para a frente e resistir ao olhar clíni- 
co de profissionais (13).

O outro estudo mostra que a utilização de fotografias e narrativas dá ênfase ao indivíduo e não à doença, em que uma maior compreensão empática da experiência da doença mental permite aos enfermeiros proporcionar uma melhor qualidade de cuidado e otimizar os resultados de saúde e qualidade de vida para uma população de clientes desafiadoras à profissão (14).

Os resultados dos estudos mostram o meio em que a utilização da fotografia na saúde começou a ser utilizada, na saúde mental. Contudo, os anos de publicação aqui evidenciados apontam para uma recente utilização desta mesma possibilidade de cuidar, no mesmo contexto em que seu uso foi iniciado.

$\mathrm{O}$ artigo de título "Cuidados de enfermagem e segurança do paciente: visualizando a organização, acondicionamento e distribuição de medicamentos com método de pesquisa fotográfica" teve como objetivo descrever os achados selecionados de relatórios de enfermeiros e acadêmicos de enfermagem acerca da organização, distribuição e acondicionamento de medicações, numa unidade de clínica médica de um hospital público de ensino, no Brasil (15).

Trata-se de um estudo diferenciado do uso da fotografia, mas no mesmo contexto da busca pela qualificação da assistência de enfermagem, ao analisar a organização de medicações utilizadas em assistência hospitalar, possibilitando uso não apenas da assistência direta ao paciente, mas os resultados indiretos desta atuação.

Com o estudo, verifica-se que a fotografia foi útil para mostrar as implicações indiretas da assistência de enfermagem, que vão além das intervenções físicas ao paciente. Além disso, foi possível verificar a diversidade de atividades do enfermeiro, não apenas do cenário hospitalar, onde é vista a necessidade de ações de gerenciamento e toda sua responsabilidade na assistência direta.

Aponta-se, portanto, para a possibilidade desses profissionais tem de utilizar este método em suas práticas, perpassando desde o registro de fatos às intervenções por ele possíveis.

Os estudos "The importance of wound documentation and classification" e "Critical care extra. Photography in nursing" tiveram como objetivo o emprego da fotografia no cuidado de enfermagem com feridas $(16,17)$.

O primeiro relata a importância da documentação do acompanhamento de pacientes com feridas (15) e o segundo descreve a utilização da fotografia como um procedimento no controle de tratamento de feridas (16). É de comum acordo que a fotografia fornece uma imagem detalhada da ferida e um registro permanente. As imagens sucessivas podem ser comparadas com a finalidade de detectar a melhoria ou deterioração da ferida (16).

Os benefícios da documentação de fotos de feridas incluem a capacidade de monitorar a cicatrização de forma precisa, consistente e com o minímo desconforto do paciente; o reforço da comunicação entre os prestadores de cuidados de saúde e destes com os pacientes; a utilização como uma ferramenta para educar os funcionários sobre cuidado apropriado da ferida; e como prova de cuidado para o reembolso do seguro. Outra virtude apontada é que os pacientes podem ser motivados por melhorias apontadas pelas imagens da ferida semana a semana. Isto pode funcionar como reforço positivo e ajudar o paciente a motivar-se a participar do plano de cuidados (15).

O estudo intitulado "A nurse-led intensive care after-care programme - development, experiences and preliminary evaluation" traz como objetivo estabelecer o cuidado intensivo após um programa de tratamento e realizar uma avaliação preliminar do serviço de acompanhamento a partir das perspectivas dos pacientes e familiares (18).

Este estudo mostra que no desenvolvimento e na criação de uma assistência de enfermagem liderada pelo intensivo programa 
de pós-tratamento é viável o uso de recursos como a fotografia, o que fora recebido polsitivamente pelos pacientes e seus familiares, significando melhoria na evolução do tratamento do cliente.

\section{DISCUSSÃO E CONCLUSÃO}

Observa-se que a fotografia tem sua aplicação possível em diversos contextos da atenção em enfermagem.

Verifica-se sua utilização por enfermeiros nos centros especializados de tratamento de feridas em diversos países (19), onde a documentação de feridas tornou-se mais importante nos últimos dez anos. A avaliação da ferida proporciona uma situação de base contra a qual um plano de cuidados do paciente pode ser avaliado (16).

Nesse contexto, os estudos concluem que os benefícios da documentação de feridas incluem a capacidade de monitorar a cicatrização de forma precisa, minimiza o desconforto do paciente, reforço da comunicação entre os prestadores de cuidados de saúde (17). A fotografia, em conjunto com as anotações de enfermagem, fornece uma forma boa de documentação da ferida e pode fornecer evidência clara se for necessário para os casos legais (16).

No contexto da saúde mental, é raro ouvir profissionais de saúde falar ou escrever sobre o uso de fotografias para avaliar problemas psicosocio-espirituais do cliente no cuidado diário. Além disso, há preocupações de que os prestadores de cuidados de saúde podem invadir a privacidade de um cliente por meio do uso de câmeras. Em alguns casos, um cliente pode, voluntariamente, compartilhar fotografias de infância ou de sua família. Essas fotos podem se tornar uma ferramenta objetiva de diagnóstico de enfermagem, não apenas por descrever aspectos físicos, mas também os aspectos psico-socio-espirituais do cliente (9). Mesmo sendo esse o contexto de início do uso de fotografias na saúde, visualizam-se poucos estudo que relatam estas experiências atualmente, mesmo que tenha sido evidenciada a utilidade e importância da foto no cuidados a esses pacientes.

Ser capaz de revelar a sua emoção através das palavras, seja verbal ou escrita, pode ser alívio de tensões (20). Através da combinação de fotografia e narrativa, é possível melhorar a qualidade do cuidado e da pesquisa, possibilitando o conhecimento, o desenvolvimento da teoria e o estabelecimento de um ambiente adequado para a mudança (14). Os estudos evidenciaram que as fotografias apresentam o que os participantes vêem como importante, pois eles iluminam valores e desejos pessoais.

Como uma intervenção de enfermagem, a foto fornece um meio para o enfermeiro estruturar os passos que os pacientes fazem em seu processo de reabilitação. Desta forma, a fotografia é uma forma de orientar, cuidar e apoiar às demandas dos pacientes e seu mundo da vida (13).

Com base nos estudos analisados, verificou-se que a fotografia também leva à uma conscientização e autoavaliação em relação ao real e cotidiano contexto do trabalho diário, que muitas vezes passa a ser automatizado. Com o uso de abordagem restaurativa para a fotografia digital, a elucidação da foto, e a narrativa da foto, podem-se identificar como tratamos um ao outro e os locais que compartilhamos; aspectos preocupantes em relação à segurança, bem como algumas práticas que poderiam ser reforçadas para fortalecer a segurança para profissionais e pacientes. Como por exemplo, o uso da imagem na identificação de fatores os quais podem levar a erros subsequentes na preparação e administração de medicamentos por enfermeiros e acadêmicos de enfermagem que são responsáveis pela organização, distribuição e acondicionamento de medicações (16).

O conteúdo extraído de material fotográfico permite a compreensão e reflexão mais aprofundada sobre o objeto de estudo, per- 
mitindo o cuidado integral e holístico do cliente.

A fotografia mostra-se como importante ferramenta de prática em enfermagem, não apenas de modo técnico, como se evidencia em sua larga utilização no tratamento de feridas, mas ainda em outros aspectos, como a organização do processo de trabalho, o registro de sentimentos e percepções de clientes e acompanhantes, fato este às vezes não visualizado pela comunicação verbal.

A quantidade de informações que podem ser obtidas por meio dos registros visuais é inúmera e possibilita ao enfermeiro planejar sua assistência, identificar as necessidades prioritárias do paciente ou do processo de trabalho de modo geral e colaboram para melhor utilização dos recursos envolvidos. Além disso, serve de registro da evolução de uma assistência prestada, podendo ser utilizada como referência para posteriores práticas de um mesmo contexto.

A enfermagem ainda se apresenta pouco na utilização deste meio de cuidar e, apesar de o emprego de recursos visuais em pesquisas científicas ter ampliado nas últimas décadas, este trabalho revela a precariedade de publicações direcionadas especificamente a essa temática, que ainda são escassas. Dos artigos que foram submetidos à análise apenas um é de origem brasileira, indicando a importância de implementar estudos sobre a documentação fotográfica nas instituições de saúde brasileiras.

Concluímos, a partir dos estudos que intentaram relatar o uso da fotografia em cuidados de enfermagem, que ainda são poucas as práticas assistenciais deste profissional utilizando essa tecnologia.

Sugerimos que, além do aprofundamento do tema na prática, estes resultados sejam mais divulgados a fim de compartilhar ações e, desta forma, contribuir para um cuidado diferenciado.

\section{REFERÊNCIAS}

1. Prado ML, Rocha PK, Backes VMS, Reibnitz KS, Waterkemper R, Gomes DC. Produção de conhecimento de um curso de mestrado em enfermagem no Brasil. Cienc. enferm. 2011; XVIII(3): 43-50.

2. Reus LH, Tittoni J. A visibilidade do trabalho de enfermagem no centro cirúrgico por meio da fotografia. Interface. 2012; 16(41): 485-500.

3. Hochman G, Mello MTB, Santos PRE. A malária em foto: imagens de campanhas e ações no Brasil da primeira metade do século XX. Hist Cienc Saude Manguinhos. 2002; 9 Suppl: 233-73.

4. Dubois P. O ato fotográfico e outros ensaios. 12 ed. Campinas (SP): Papirus; 2009.

5. Borges MD, Aranha JM, Sabino J. A fotografia de natureza como instrumento para educação ambiental. Ciênc Educ. 2010; 16(1): 149-61.

6. Faria NGF. Fotografia digital de feridas: desenvolvimento e avaliação de curso online para enfermeiros. [Tese (Doutorado]. [São Paulo (SP)]: Escola de Enfermagem da Universidade de São Paulo; 2010. $132 \mathrm{p}$.

7. Melleiro MM, Gualda DMR. A abordagem fotoetnográfica na avaliação de serviços de saúde e de enfermagem. Texto contexto-enferm. 2006; 15(1): 82-8.

8. Faria NGF, Peres HHC. Análise da produção científica sobre documentações fotográficas de feridas em enfermagem. Rev. Eletr. Enf. [Internet]. 2009 [cited 2012 Sep 15]; 11(3): 704-11. Disponível em: http://www.fen.ufg.br/fen_revista/ v11/n3/pdf/v11n3a31.pdf

9. Felicilda RF. Pictures of a client's silent distress. Creat Nurs. 2011; 17(4): 180-3.

10. Mendes KDS, Silveira RCCP, Galvão CM. 
Revisão integrativa: método de pesquisa para a incorporação de evidências na saúde e na enfermagem. Texto contextoenferm. 2008; 17(4): 758-64.

11. Broome ME. Integrative literature reviews for the development of concepts. In: Rodgers BL, Knafl KA. Concept development in nursing: foundations, techniques and applications. Philadelphia: WB Saunders Company; 2000. p. 231-50.

12. Whittemore R, Knafl K. The integrative review: updated methodology. J Adv Nurs. 2005; 52(5): 546-53.

13. Sitvast JE, Widdershoven GAM, Abma TA. Moral learning in psychiatric rehabilitation. Nurs Ethics. 2011; 18(4): 58395.

14. Fleming J, Mahoney J, Carlson E, Engebretson J. An ethnographic approach to interpreting a mental illness photovoice exhibit. Arch Psychiatr Nurs. 2009; 23(1): 16-24.

15. Raduenz AC, Hoffmann P, Radunz V, Dal Sasso GTM, Maliska ICA, Mark PB.
Cuidados de enfermagem e segurança do paciente: visualizando a organização, acondicionamento e distribuição de medicamentos com método de pesquisa fotográfica. Rev Lat Am Enfermagem. 2010. 18(6): 1045-54.

16. Russell L. The importance of wound documentation and classification. Br J Nurs. 1999; 8(20): 1342-54.

17. Krainski M. Photography in Nursing. Am J Nurs. 1998; 98(9): 16BB,16DD-16EE.

18. Samuelson KA, Corrigan I. A nurse-led intensive care after-care programme development, experiences and preliminary evaluation. Nurs Crit Care. 2009; 14(5): 254-63.

19. Fischetti LF, Paguio EC. Alt-White AC. Digitized images of wounds: a nursing practice innovation. Nurs Clin North Am. 2000; 35(2): 541-50.

20. Seaward BL. Managing stress: a creative journal. $4^{\text {a }}$ ed. Sudbury, MA: Jones \& Bartlett Learning; 2011. 